MC/TH 95/22

\title{
Determination of the pion-nucleon coupling constant from QCD sum rules
}

\author{
Michael C. Birse and Boris Krippa* \\ Theoretical Physics Group, Department of Physics and Astronomy \\ University of Manchester, M13 9PL, UK
}

\begin{abstract}
We evaluate the $\pi N$ coupling constant using a QCD sum rule based on the pion-to-vacuum matrix element of the correlator of two interpolating nucleon fields. The part of the correlator with Dirac structure $\not k \gamma_{5}$ is used, keeping all terms up to dimension 5 in the OPE and including continuum contributions on the phenomenological side. The ratio of this sum rule to the nucleon sum rule involving condensates of odd dimension yields stable results with values of $g_{\pi N}$ in the range $12 \pm 5$. The sources of uncertainty are discussed.
\end{abstract}

Understanding hadron interactions from first principles is one of the main goals of Quantum Chromodynamics (QCD). Since the solution of QCD for hadron interactions at low energies is still far off it is useful to consider tackle problems of hadron dynamics with approaches that lie as close as possible to QCD. One of them, the method of QCD sum rules [1], has proved to be a very powerful tool to extract information about hadron properties.

\footnotetext{
*Permanent address: Institute for Nuclear Research of the Russian Academy of Science, Moscow Region 117312, Russia.
} 
We present here a sum rule analysis of the $\pi N$ coupling constant $g_{\pi N}$, which is one of the most important parameters in hadron physics. This quantity has previously been examined within the framework of QCD sum rules in Refs. [3, [3]. Reinders, Rubinstein and Yazaki [2] worked with two different sum rules for $g_{\pi N}$, one obtained from a correlator of three interpolating fields, and one based on the pion-to-vacuum matrix element of the correlator of two interpolating nucleon fields, $\eta$ :

$$
\left\langle 0|T\{\eta(x) \bar{\eta}(0)\}| \pi^{a}(k)\right\rangle,
$$

However those authors included only the leading term of operator product expansion (OPE) and neglected continuum contributions. Shiomi and Hatsuda [3] developed the sum rule based on the part of this two-point correlation function with Dirac structure $\gamma_{5}$. They calculated $g_{\pi N}$ in the soft-pion limit, including condensates up to dimension 7 in the OPE, radiative corrections and a perturbative estimate of continuum contributions.

The method based on the two-point correlator (1) has a significant advantage in that it can be used for low values of the momentum transfer to the nucleon. In contrast the OPE of the three-point correlator is valid only for large spacelike meson momenta and so a determination of the coupling constant requires an extrapolation to zero momentum (see, for example 四). This procedure is dangerous because of the higher-order terms that have been omitted from the OPE. The contributions of these terms give rise to corrections that are proportional to large inverse powers of the meson momentum $k$, making the extrapolation of a truncated OPE unreliable. Estimates of the coupling constant from the coefficient of $1 / k^{2}$ determined at large $k^{2}$, as in Refs. [5.2], cannot distinguish the meson pole term from the contributions of higher-mass states in the same meson channel.

In the soft-pion limit the OPE for the $\gamma_{5}$ part of the two-point correlator for $g_{\pi N}$ has exactly the same form as that for the nucleon sum rule [6.7] involving condensates of odd dimension, up a factor of $1 / f_{\pi}$ [2].3]. If continuum corrections are neglected, the ratio of these two sum rules has the form of the Goldberger-Treiman relation with $g_{A}=1$ [2]. Shiomi and Hatsuda [3] showed that this continues to hold for the higher-dimension terms in the OPE, 
provided that the continuum thresholds are taken to be the same for both sum rules. Using different thresholds in the two sum rules they were able to get around this problem with the implied value of $g_{A}$.

However, using the usual soft-pion theorem [8], the correlator (1) can be expressed as

$$
-\frac{i}{f_{\pi}}\left\langle 0\left|\left[Q_{5}^{a}, T\left(\eta(x), \eta^{\dagger}(0)\right)\right]\right| 0\right\rangle=\frac{i}{2 f_{\pi}}\left\{\gamma_{5} \tau^{a},\left\langle 0\left|T\left(\eta(x), \eta^{\dagger}(0)\right)\right| 0\right\rangle\right\}
$$

where $Q_{5}^{a}$ is the axial charge and we have made use of the transformation properties of the interpolating field under axial rotations [9], $\left[Q_{5}^{a}, \eta\right]=-\frac{1}{2} \gamma_{5} \tau^{a} \eta$. The anticommutator with $\gamma_{5}$ picks out the part of the two-point correlator proportional to the unit Dirac matrix. The phenomenological side of the resulting sum rule is thus $i \gamma_{5} / f_{\pi}$ times the corresponding expression for the odd-condensate nucleon sum rule. This matches exactly with the structure found for the OPE side in Refs. [2, 3].

The soft-pion limit for the $\gamma_{5}$ piece of the correlator (1) thus yields a sum rule for $M_{N} / f_{\pi}=g_{\pi N} / g_{A}$. The value for $g_{\pi N}$ determined from such a rum rule follows from the odd-condensate sum rule for the nucleon mass and the Goldberger-Treiman relation (or an approximation to it taking $g_{A}=1$ ). The sum rule can be thought of as just a chiral rotation of the odd-condensate nucleon sum rule and not an independent determination of $g_{\pi N}$. By considering terms beyond the soft-pion limit, we obtain here a value for $g_{\pi N}$ that is not simply a consequence of chiral symmetry. The sum rule we use is thus analogous to that for the $\pi N \Delta$ coupling [2] and the approach can be applied to calculations of other meson-baryon couplings.

Moreover, a potentially important piece of the phenomenological side of the sum rule for $g_{\pi N}$ has been omitted in both calculations. This term corresponds to transitions of where a ground-state nucleon absorbs the pion and is excited into the continuum. Since it is not suppressed by the Borel transformation such a term should be included in a consistent sumrule analysis, as pointed out long ago [7, 10] and stressed recently by Ioffe [11, [12]. In the soft-pion limit, such terms generate contact interactions where the pion couples directly to the nucleon field, $\left\langle N(p)\left|\bar{\eta}_{n}(0)\right| \pi(k)\right\rangle$, and which are essential if the correct soft-pion limit 
is to be obtained. Although the need for these terms is particularly clear if pseudovector $\pi N$ coupling is used (see for example [13]), they should also be included for pseudoscalar coupling. Their omission in Refs. [2, [3] can explain why the correct Goldberger-Treiman relation was not found there. Indeed, as the authors of [3] point out, a quick estimate of these unsuppressed $N^{*}$ contributions suggests that they could be as large as 25\%: enough to remove the discrepancy with the Goldberger-Treiman relation.

Here we start from the two-point correlator (1) just discussed, but instead of the piece with with Dirac structure $\gamma_{5}$ considered in Refs. [2, 3] we work with the structure $\not k \gamma_{5}$, where $k$ is the pion momentum. We work here to leading order in a chiral expansion, neglecting higher-order terms in the pion momentum or current quark mass.

We consider the two-point correlation function

$$
\Pi(p)=i \int d^{4} x \exp (i p \cdot x)\left\langle 0\left|T\left\{\eta_{p}(x) \bar{\eta}_{n}(0)\right\}\right| \pi^{+}(k)\right\rangle
$$

where we use the Ioffe interpolating field [6] for the proton,

$$
\eta_{p}(x)=\epsilon_{a b c}\left[u^{a}(x)^{T} C \gamma_{\mu} u^{b}(x)\right] \gamma_{5} \gamma^{\mu} d^{c}(x)
$$

where $a, b, c$ are the colour indices and $C$ is the charge conjugation matrix. (The corresponding neutron field $\eta_{n}$ is obtained by interchanging $u$ and $d$ quark fields.) More general choices of interpolating field are possible, as discussed in detail by Leinweber [14]. For the odd-condensate nucleon sum rule, which we make use of in our determination of $g_{\pi N}$, it turns out that the Ioffe field is close to the optimal one as determined in Ref. [14] and so we do not consider more general fields here.

In the deeply Euclidean region ( $p^{2}$ large and negative) the OPE of the product of two interpolating fields takes the following general form

$$
i \int d^{4} x \exp (i p \cdot x) T\left\{\eta_{p}(x) \bar{\eta}_{n}(0)\right\}=\sum_{n} C_{n}(p) O_{n}
$$

where $C_{n}(p)$ are the Wilson coefficients and $O_{n}$ are local operators constructed out of quark and gluon fields (all renormalised at some scale $\mu$ ). Using this OPE in the correlator (3), we 
find that only operators of odd dimension contribute. The leading term in this expansion involves operators with dimension 3 and has the form

$$
\Pi_{3}(p, k)=-\frac{1}{2 \pi^{2}} p^{2} \ln \left(-p^{2}\right)\left\langle 0\left|\bar{d} \gamma^{\alpha} \gamma_{5} u\right| \pi^{+}(k)\right\rangle \gamma_{\alpha} \gamma_{5}+\cdots
$$

where terms that do not contribute to the Dirac structure of interest, $\not k \gamma_{5}$, have been suppressed. The matrix element here is just the usual one for pion decay:

$$
\left\langle 0\left|\bar{d} \gamma^{\alpha} \gamma_{5} u\right| \pi^{+}(k)\right\rangle=i \sqrt{2} f_{\pi} k^{\alpha}
$$

where $f_{\pi}=93 \mathrm{MeV}$ is the pion decay constant.

At dimension 5 the only relevant contribution arises from the second-order term in the covariant expansion of the nonlocal operator $\bar{d}(0) \gamma^{\alpha} \gamma_{5} u(x)$. It has the form

$$
\Pi_{5}(p)=\frac{5}{9 \pi^{2}} \ln \left(-p^{2}\right)\left\langle 0\left|\bar{d} \gamma^{\alpha} \gamma_{5} D^{2} u\right| \pi^{+}(k)\right\rangle \gamma_{\alpha} \gamma_{5}+\cdots
$$

Up to corrections of higher order in the current mass, this can easily be re-expressed in terms of a mixed quark-gluon condensate

$$
\left\langle 0\left|\bar{d} \gamma^{\alpha} \gamma_{5} D^{2} u\right| \pi^{+}(k)\right\rangle=\frac{g_{s}}{2}\left\langle 0\left|\bar{d} \gamma^{\alpha} \gamma_{5} \sigma_{\mu \nu} G^{\mu \nu} u\right| \pi^{+}(k)\right\rangle+\mathcal{O}\left(m_{c}^{2}\right)
$$

Some further manipulation allows one to rewrite this in the form

$$
\left\langle 0\left|\bar{d} \gamma^{\alpha} \gamma_{5} D^{2} u\right| \pi^{+}(k)\right\rangle=-g_{s}\left(\left\langle 0\left|\bar{d} \widetilde{G}^{\alpha \mu} \gamma_{\mu} u\right| \pi^{+}(k)\right\rangle-i g_{s}\left\langle 0\left|\bar{d} G^{\mu \alpha} \gamma_{\mu} \gamma_{5} u\right| \pi^{+}(k)\right\rangle\right)
$$

where $\widetilde{G}_{\mu \nu}=\frac{1}{2} \epsilon_{\mu \nu \rho \sigma} G^{\rho \sigma}$. (We use the convention $\epsilon^{0123}=+1$.) The second term in this expression is of higher order in the chiral expansion (see Ref. [15] for details) and so we neglect it here.

The first matrix element in (10) was extracted by Novikov et al. 115 from two QCD sum rules for the pion. They expressed it in the form

$$
g_{s}\left\langle 0\left|\bar{d} \widetilde{G}^{\alpha \mu} \gamma_{\mu} u\right| \pi^{+}(k)\right\rangle=\sqrt{2} i \delta^{2} f_{\pi} k^{\alpha}
$$


and obtained $\delta^{2}=(0.20 \pm 0.02) \mathrm{GeV}^{2}$. A crucial contribution in both of their sum rules is the four-quark condensate, $\alpha_{s}\left\langle 0\left|(\bar{q} q)^{2}\right| 0\right\rangle$. Novikov et al. [15] used the factorisation approximation for this quantity but direct determinations of it from other sum rules suggest significantly larger values [16 18, at least 2-3 times bigger than those obtained from factorisation. These give correspondingly larger values for $\delta^{2}$, a point we shall come back to in the discussion of our results below.

As an estimate of the importance of higher dimension condensates, we have also calculated the contribution of what we hope is the most important dimension-7 operator in the OPE. This is a mixed quark-gluon condensate, which we evaluate in the factorised approximation. Keeping only this contribution explicitly, the dimension-7 piece of the correlator is

$$
\Pi_{7}(p)=-\frac{1}{12 p^{2}}\left\langle 0\left|\bar{d} \gamma^{\alpha} \gamma_{5} u\right| \pi^{+}(k)\right\rangle\left\langle 0\left|\frac{\alpha_{s}}{\pi} G^{2}\right| 0\right\rangle \gamma_{\alpha} \gamma_{5}+\cdots
$$

where $\left\langle 0\left|\frac{\alpha_{s}}{\pi} G^{2}\right| 0\right\rangle$ is the gluon condensate in vacuum.

On the phenomenological side, the term of interest in the correlator (33) is the one with a double pole at the nucleon mass, since this contains the $\pi N$ coupling constant. However the nucleon interpolating field does not just create ground-state nucleons; there are also continuum contributions which cannot be ignored. The continuum-to-continuum pieces are modelled in the usual manner, in terms of the spectral density associated with the imaginary part of the OPE expression for the correlator. This continuum is assumed to start at some threshold $S_{\pi N}$. After Borel transformation, it can be taken over to the OPE side of the sum rule where it modifies the coefficients of the terms involving $\ln \left(-p^{2}\right)$. In addition one must

\footnotetext{
${ }^{1}$ There is a potential sign ambiguity in using the result of Ref. 15] since they do not specify their convention for $\epsilon^{0123}$. However we have checked that our coefficient of $x^{2}$ in the expansion of $\left\langle 0\left|\bar{d}(0) \gamma^{\alpha} \gamma_{5} u(x)\right| \pi^{+}\right\rangle$(which should be independent of convention) agrees in both sign and magnitude with that of the corresponding term in the expansion of $\langle 0|u(x) \bar{u}(0)| 0\rangle_{A}$ given in Refs. [10,12].
} 
include nucleon-to-continuum terms since Borel transformation does not suppress these with respect to the double-pole term [7, 10 12]. To first order in $k$, the correlator has the form

$$
\Pi(p)=i \sqrt{2} \not k \gamma_{5}\left[\frac{\lambda_{N}^{2} M_{N} g_{\pi N}}{\left(p^{2}-M_{N}^{2}\right)^{2}}+\int_{W^{2}}^{\infty} d s b(s) \frac{1}{s-M_{N}^{2}}\left(\frac{1}{p^{2}-M_{N}^{2}}+\frac{a(s)}{s-p^{2}}\right)\right]+\cdots,
$$

where the continuum-continuum terms (and terms with other Dirac structures) have not been written out. Here $\lambda_{N}$ is the strength with which the interpolating field couples to the nucleon:

$$
\left\langle 0\left|\eta_{N}(0)\right| N(p)\right\rangle=\lambda_{N} u(\mathrm{p})
$$

Note that the strength of the $\not k \gamma_{5}$ piece of the double-pole term is the same for both pseudoscalar and pseudovector $\pi N$ coupling.

Equating the OPE and phenomenological expressions for the correlator (3) and Borel transforming [1], we get the sum rule

$$
\frac{1}{2 \pi^{2}} M^{4} E_{2}(x)+\frac{5}{9 \pi^{2}} M^{2} E_{1}(x) \delta^{2}+\frac{1}{12} E_{0}(x)\left\langle 0\left|\frac{\alpha_{s}}{\pi} G^{2}\right| 0\right\rangle=\left(\frac{\lambda_{N}^{2} M_{N} g_{\pi N}}{f_{\pi} M^{2}}+A\right) \exp \left(-M_{N}^{2} / M^{2}\right),
$$

where $M$ is the Borel mass and $E_{n}(x)=1-\left(1+x+\ldots+\frac{x^{n}}{n !}\right) e^{-x}$ with $x=\frac{S_{\pi N}}{M^{2}}$. The second term on the r.h.s. of this sum rule, involving the undetermined constant $A$, is the Borel transform of the nucleon pole term of the nucleon-to-continuum piece in (13). It contains the same exponential as the nucleon double-pole term and so cannot be ignored. The second nucleon-to-continuum term in (13) leads to a term that is suppressed by an exponential involving the masses of states in the continuum. It is thus typically a factor of 3-4 smaller than the term included in (15). Provided that the first of these mixed terms is a reasonably small correction to the sum rule, it should be safe to neglect the second, as discussed by Ioffe [1],12].

We now turn to the numerical analysis of this sum rule. First, one should get rid of the unknown constant $A$. Multiplying the sum rule by $M^{2} \exp M_{N}^{2} / M^{2}$, we see that the r.h.s. becomes a linear function of $M^{2}$. By acting on this form of the sum rule with 
$\left(1-M^{2} \partial / \partial M^{2}\right)$ [7] (or equivalently by fitting a straight line to the l.h.s. and extrapolating to $M^{2}=0$ [10]) we in principle can determine the value of $g_{\pi N}$. However we are unable to find a region of Borel mass in which the l.h.s. is approximately a linear function of $M^{2}$, and hence there is no region of stability for the extracted $g_{\pi N}$.

This lack of stability is similar to the situation for the nucleon sum rules, where two sum rules can be derived [6] (involving either odd or even dimension operators) but neither shows good stability. Nonetheless the ratio of these leads to a more stable expression for the nucleon mass. We have therefore taken the ratio of our sum rule (15) to the nucleon sum rules. We obtain the most stable results from the ratio to the odd-dimension sum rule,

$$
-\frac{1}{4 \pi^{2}} M^{4} E_{1}\left(x_{N}\right)\langle 0|\bar{q} q| 0\rangle+\frac{1}{24}\langle 0|\bar{q} q| 0\rangle\left\langle 0\left|\frac{\alpha_{s}}{\pi} G^{2}\right| 0\right\rangle=\lambda_{N}^{2} M_{N} \exp \left(-M_{N}^{2} / M^{2}\right),
$$

and so we present here only the results for that case. Taking such a ratio also has the advantage of eliminating the experimentally undetermined strength $\lambda_{N}$ from the sum rules. Note that we have allowed for a different continuum threshold $S_{N}$ in the nucleon sum rule and have defined $x_{N}=S_{N} / M^{2}$.

The ratio of the sum rules (15) and (16) can be written in the form

$$
f_{\pi} \frac{\frac{1}{2 \pi^{2}} M^{6} E_{2}(x)+\frac{5}{9 \pi^{2}} M^{4} E_{1}(x) \delta^{2}+\frac{1}{12} M^{2} E_{0}(x)\left\langle 0\left|\frac{\alpha_{s}}{\pi} G^{2}\right| 0\right\rangle}{-\frac{1}{4 \pi^{2}} M^{4} E_{1}\left(x_{N}\right)\langle 0|\bar{q} q| 0\rangle+\frac{1}{24}\langle 0|\bar{q} q| 0\rangle\left\langle 0\left|\frac{\alpha_{s}}{\pi} G^{2}\right| 0\right\rangle}=g_{\pi N}+A^{\prime} M^{2},
$$

and the method discussed above used to eliminate the unknown mixed nucleon-to-continuum term, $A^{\prime} M^{2}\left(A^{\prime}=A f_{\pi} / \lambda_{N}^{2} M_{N}\right)$. In Fig. 1 we show results for $g_{\pi N}$ as a function of $M^{2}$, for typical values of the condensates and thresholds: $\langle 0|\bar{q} q| 0\rangle=-(0.245 \mathrm{GeV})^{3},\left\langle 0\left|\frac{\alpha_{s}}{\pi} G^{2}\right| 0\right\rangle \simeq$ $0.012 \mathrm{GeV}^{4}, \delta^{2}=0.35 \mathrm{GeV}^{2}, S_{N}=2.5 \mathrm{GeV}$ and $S_{\pi_{N}}=2.15 \mathrm{GeV}$. Stable values of $g_{\pi N} \simeq$ 11.7 are found over a region $M^{2} \simeq 0.8-1.8 \mathrm{GeV}^{2}$. Corrections due to the $A^{\prime} M^{2}$ term are small, at most $5 \%$. The second such term in (13) is expected to be smaller by a factor of $3-4$, and so we are justified in neglecting it.

The threshold $S_{\pi N}$ has been adjusted to give stability for $M^{2}$ around $1 \mathrm{GeV}^{2}$, since one may hope that in this region the Borel transformed sum rule is not too sensitive to the approximations that have been made on both the OPE and phenomenological sides of the 
sum rule. The existence of a window of stability provides a check on the consistency of this assumption. We also demand that the thresholds $S_{N}$ and $S_{\pi N}$ should lie significantly above this window so that the continuum is not too heavily weighted in the Borel transform. We find that the window of stability moves rapidly upwards as $S_{\pi N}$ is increased for fixed $S_{N}$. For the typical parameter values above, only the region $2.05 \mathrm{GeV}^{2} \leq S_{\pi N} \leq 2.22 \mathrm{GeV}^{2}$ satisfies these requirements. The value of $g_{\pi N}$ varies by at most \pm 0.2 over this region.

As a further check on our results, we have examined whether the individual sum rules (15) and (16) satisfy the criteria suggested by Leinweber [14]. We find that the highest dimension condensates contribute less that $10 \%$ of the OPE to both sum rules for $M^{2}>0.8$ $\mathrm{GeV}^{2}$. The continuum forms about $40 \%$ of the phenomenological side of the differentiated version of the $g_{\pi N}$ sum rule (15) for $M^{2}$ up to $1.4 \mathrm{GeV}^{2}$, the point at which the continuum reaches $50 \%$ of the odd-condensate sum rule (16). The region $M^{2} \simeq 0.8-1.4 \mathrm{GeV}^{2}$ thus provides a window within which our results are both stable with respect to the Borel mass and not too sensitive to our approximations.

We have examined the dependence of our results on the other input parameters. Variation of the threshold in the nucleon sum rule $S_{N}$ from 2.2 to $2.8 \mathrm{GeV}^{2}$, readjusting $S_{\pi N}$ to maintain stability, changes $g_{\pi N}$ by \pm 0.2 . To estimate the sensitivity of our sum rule to the contributions of dimension-7 condensates and to uncertainties in the gluon condensate, we have varied the dimension-7 term in (15) between zero and twice its standard value. Our results for $g_{\pi N}$ change by \pm 0.5 over this range.

One of the most important input parameters in our sum rule is the matrix element $\delta^{2}$, defined by (11). As already mentioned, this parameter was extracted by Novikov et al. 15] from an analysis of two sum rules for the pion. Their results depend crucially on the four-quark condensate, $\alpha_{s}\left\langle 0\left|(\bar{q} q)^{2}\right| 0\right\rangle$, for which they made the factorisation approximation and took a value of about $2 \times 10^{-4} \mathrm{GeV}^{6}$. With this input, both of their sum rules yield consistent results for $\delta^{2}$ in the region $0.20 \pm 0.02 \mathrm{GeV}^{2}$. However, sum rules analyses of $\tau$ decay and $e^{+} e^{-}$annihilation into hadrons lead to significantly larger values of the fourquark condensate (see 16 18 and references therein), in the range $(4-6) \times 10^{-4} \mathrm{GeV}^{6}$. 
Using these in the sum rules of Ref. [15] leads to values for $\delta^{2}$ ranging from 0.28 to 0.45 , although the two sum rules do not then give consistent results. As a conservative estimate of the uncertainty in $\delta^{2}$ we have considered the range 0.20 to $0.45 \mathrm{GeV}^{2}$. The corresponding variation in $g_{\pi N}$ is \pm 2 , when the other parameters are held at their values above and $S_{\pi N}$ is changed to keep the window of stability around $1 \mathrm{GeV}^{2}$.

A second significant source of uncertainty is the quark condensate $\langle 0|\bar{q} q| 0\rangle$ which appears in the odd-dimension sum rule for the nucleon. "Standard" values for this lie in the range $-(0.21 \mathrm{GeV})^{3}$ and $-(0.26 \mathrm{GeV})^{3}$. The values of the nucleon mass determined from sum rules [6] are strongly correlated with this condensate. There is also a weaker correlation with the chosen value of the threshold $S_{N}$. Since we are dividing by $M_{N}$ in the ratio (17), our results are rather sensitive to the value of this condensate. One would like to use values of $\langle 0|\bar{q} q| 0\rangle$ and $S_{N}$ that give the nucleon mass correctly, but the ratio of the odd and even dimension nucleon sum rules does not yield completely stable results for $M_{N}$. The best we can do is to rule out values of $-\langle 0|\bar{q} q| 0\rangle$ below $(0.23 \mathrm{GeV})^{3}$ since they cannot reproduce the nucleon mass within the region of Borel mass and threshold that we consider. Varying the quark condensate between $-(0.23 \mathrm{GeV})^{3}$ and $-(0.26 \mathrm{GeV})^{3}$, we find that $g_{\pi N}$ changes by $\pm 2$.

Our final result for the $\pi N$ coupling constant is thus $g_{\pi N}=12 \pm 5$, where the uncertainty is dominated by $\delta^{2}$ and $\langle 0|\bar{q} q| 0\rangle$. This is to be compared with values deduced from $N N$ and $\pi N$ scattering. For many years the accepted value was $g_{\pi N}=13.4$ [19 but this coupling has been the subject of some debate in recent years. More recent analyses lead to values in the range 12.7-13.6 20]. Our result is obviously consistent with any of these. The rather large uncertainty in it could be reduced if the quark condensate could be determined more precisely. In addition, the sum rules of Novikov et al. [15] should be re-examined using larger values of the four-quark condensate to try to pin down $\delta^{2}$ more exactly.

In summary, we have calculated the $\pi N$ coupling constant using a QCD sum rule based on the pion-to-vacuum matrix element of a two-point correlator of interpolating nucleon fields. This approach avoids the need for extrapolation from large spacelike meson momenta. We 
have included nucleon-to-continuum terms omitted from previous analyses. Our sum rule is based on the part of the correlator with Dirac structure $\not k \gamma_{5}$ and includes all terms up to dimension 5 in the OPE. Stable results are obtained from the ratio of this sum rule to one for the nucleon mass and the unsuppressed nucleon-to-continuum contributions are

found to be small. Contributions from higher-dimension operators and omitted continuum contributions are estimated to be small. This demonstrates the practicability of this type of sum rule for calculation of other meson-baryon couplings, whose values are at present not well determined.

\section{ACKNOWLEDGEMENTS}

We are grateful to V. Kartvelishvili for useful discussions. This work was supported by the EPSRC and PPARC. 


\section{REFERENCES}

[1] M. A. Shifman, A. I. Vainshtein and V. I. Zakharov, Nucl. Phys. B147 (1979) 385, 448.

[2] L. J. Reinders, H. Rubinstein and S. Yazaki, Phys. Reports 127 (1985) 1.

[3] H. Shiomi and T. Hatsuda, Nucl. Phys. A594 (1995) 294.

[4] T. Meissner, Phys. Rev. C52 (1995) 3386.

[5] L. J. Reinders, H. Rubinstein and S. Yazaki, Nucl. Phys. B213 (1983) 109.

[6] B. L. Ioffe, Nucl. Phys. B188 (1981) 317; B191 591(E).

[7] B. L. Ioffe and A. V. Smilga, Nucl. Phys. B232 (1984) 109.

[8] J. F. Donoghue, E. Golowich and B. R. Holstein, Dynamics of the standard model (Cambridge University Press, Cambridge, 1992).

[9] S. H. Lee, S. Cho, T. D. Cohen and D. K. Griegel, Phys. Lett. B348 (1995) 263.

[10] V. M. Belaev and Ya. I. Kogan, Phys. Lett. 136B (1983) 273.

[11] B. L. Ioffe, University of Bern preprint BUTP-94/25, hep-ph/9501319 (1995).

[12] B. L. Ioffe, ITEP preprint 62-95, hep-ph/9511401 (1995).

[13] Y. Koike, Phys. Rev. D48 (1993) 2313.

[14] D. B. Leinweber, University of Washington preprint DOE/ER/40427-17-N95, nuclth/9510051.

[15] V. A. Novikov, M. A. Shifman, A. I. Vainshtein, M. B. Voloshin and V. I. Zakharov, Nucl. Phys. B237 (1984) 525.

[16] V. G. Kartvelishvili and M. V. Margvelashvili, Z. Phys. C55 (1992) 83; V. Kartvelishvili, Phys. Lett. B287 (1992) 159.

[17] E. Braaten, S. Narison and A.Pich, Nucl. Phys. B373 (1992) 581. 
[18] S. Narison, Phys. Lett. B361 (1995) 121.

[19] D. V. Bugg, A. A. Carter and J. R. Carter, Phys. Lett. 44B (1973) 278.

[20] V. Stoks, R. Timmermans and J. J. de Swart, Phys. Rev. C47 (1993) 512;

R. A. Arndt, R. L. Workman and M. Pavan, Phys. Rev. C49 (1994) 2729;

F. Bradamante, A. Bressan, M. Lamanna and A. Martin, Phys. Lett. B343 (1995) 431;

T. E. O. Ericson et al., Phys. Rev. Lett. 75 (1995) 1046;

D. V. Bugg and R. Machleidt, Phys. Rev. C52 (1995) 1203. 


\section{FIGURE CAPTION}

Fig. 1. Dependence on the square of the Borel mass of the $\pi N$ coupling constant determined from the ratio of sum rules (17). The values of the parameters used are given in the text. The solid line shows the value of $g_{\pi N}$ corrected for the mixed continuum term $A^{\prime} M^{2}$, the dashed line the uncorrected l.h.s. of (17). 


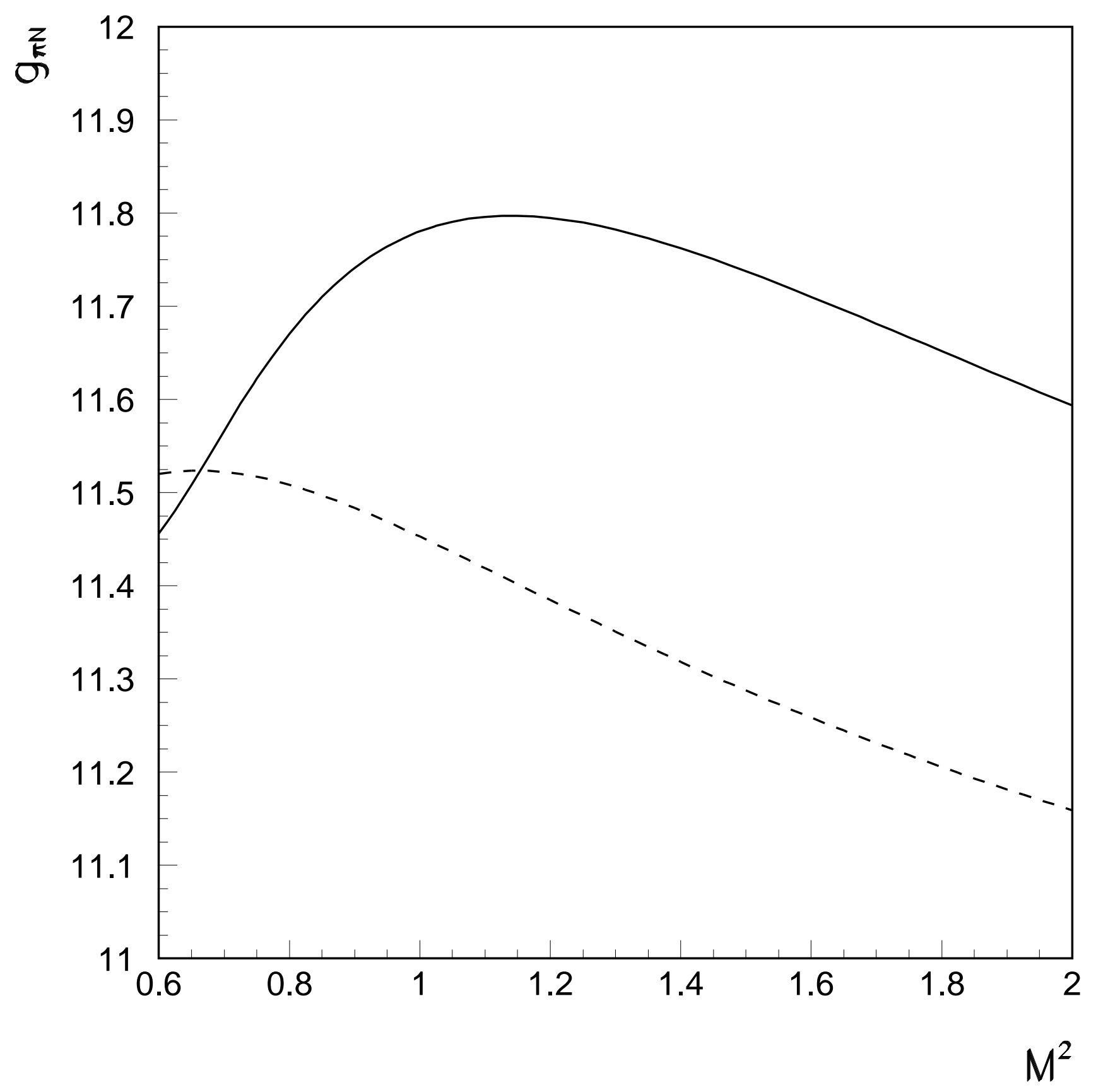

